\title{
ASp
}

la revue du GERAS

11-14 | 1996

Actes du 17e colloque du GERAS

\section{Les nouvelles technologies éducatives dans l'exploitation du scénario pédagogique}

\section{Donna Andréolle}

\section{(2) OpenEdition}

\section{Journals}

\section{Édition électronique}

URL : http://journals.openedition.org/asp/3690

DOI : 10.4000/asp.3690

ISSN : 2108-6354

\section{Éditeur}

Groupe d'étude et de recherche en anglais de spécialité

\section{Édition imprimée}

Date de publication : 1 décembre 1996

Pagination : 407-416

ISSN : 1246-8185

\section{Référence électronique}

Donna Andréolle, «Les nouvelles technologies éducatives dans l'exploitation du scénario pédagogique », ASp [En ligne], 11-14 | 1996, mis en ligne le 24 juillet 2013, consulté le 19 avril 2019. URL : http:// journals.openedition.org/asp/3690 ; DOI : 10.4000/asp.3690

Ce document a été généré automatiquement le 19 avril 2019

Tous droits réservés 


\title{
Les nouvelles technologies éducatives dans l'exploitation du scénario pédagogique
}

\author{
Donna Andreolle
}

\section{Introduction}

1 L'enseignement des langues, dont l'anglais langue de spécialité, subit actuellement une mutation due à l'explosion du progrès technologique de ces dernières années. L'apparition du CD-ROM interactif, de la nouvelle génération de laboratoire de langues, et l'arrivée d'Internet dans les établissements scolaires et universitaires ont bouleversé nos «attitudes " pédagogiques dans la salle de classe; dans certains cas, il est difficile de ne pas se laisser littéralement déborder par le déluge d'informations disponibles. De même si cette technologie favorise l'autonomie de l'apprenant, il ne faut pas confondre cette liberté d'apprendre avec l'abandon de l'apprenant devant la machine. L'enseignant plus que jamais devient le chef d'orchestre au milieu d'une multitude d'instruments qui ne feront que du bruit si l'on ne prend pas le soin de les diriger vers une finalité harmonieuse.

2 Prenons par exemple le cas (réel) d'un collègue qui a demandé à ses étudiants, sans donner aucune autre indication, de faire une recherche sur le féminisme aux États-Unis en utilisant les services du Web. Ce même collègue s'est ensuite étonné lorsque ses étudiants ont fait un exposé sur le féminisme... vu par l'extrême droite américaine ! Il est évident que sans contexte culturel et sans « orchestration » pédagogique, on ne peut pas s'attendre à ce que l'étudiant ait les connaissances nécessaires pour faire la différence entre des données factuelles et des opinions politiques. Mais si au contraire nous utilisons cette diversité d'informations, ces divergences d'opinions au service d'une pédagogie interactive qui favorise à la fois la connaissance du contexte culturel et la confrontation d'idées, nous pourrons aider l'apprenant à mieux appréhender la culture de la langue 
cible et à accroître ses compétences langagières dans une situation donnée. Le scénario pédagogique est l'outil parfait pour réaliser cet objectif triple de l'interactivité, de la culture et du développement des compétences dans la langue cible.

\section{Qu'est-ce le scénario pédagogique?}

3 Le scénario pédagogique s'inspire du format d'examen choisi pour le Diplôme de Compétence en Langue; il s'agit d'une mise en situation dans laquelle le candidat joue un rôle pour accomplir une tâche globale. Le scénario comporte cinq phases :

4 医 une première phase de réception qui permet d'évaluer la capacité à décoder, à comprendre et à analyser un ensemble de documents écrits ;

5 - une deuxième phase de réception qui permet d'évaluer la capacité à comprendre et à analyser un document sonore ou vidéo ;

6 - une troisième phase interactive de réception-production orale qui permet d'évaluer la capacité à communiquer dans une situation interactive ;

7 - une quatrième phase de production écrite qui permet d'évaluer la capacité à restituer ou à transmettre une information par écrit ;

8 - une cinquième phase interactive de production orale qui permet d'évaluer la capacité à faire une présentation organisée et structurée, à argumenter un point de vue et éventuellement à soutenir une contre-argumentation (Isani et alii 1996 : 17).

9 Tout en gardant les structures fondamentales (contenu des phases), le scénario pédagogique reste un outil plus souple, comme le montrent les exemples dans la deuxième partie de cet article. De manière générale, les principales différences entre le scénario d'examen et sa variante pédagogique sont: 医 lo les phases I, II et III peuvent être placées dans un ordre différent ;

11 - la densité des informations à recueillir dans les phases de réception peut être variable suivant le nombre de séances accordées à la réalisation de la tâche ;

12 - les deux phases d'interactivité ne sont pas des situations de face-à-face entre apprenant et enseignant, mais une activité de groupe pendant laquelle les apprenants échangent les informations ou leurs points de vue respectifs ;

13 - la phase de production écrite est faite en dehors du cours et souvent après le déroulement de la phase $\mathrm{V}$.

14 Enfin, suivant le niveau et la spécialité des apprenants pour qui le scénario pédagogique est fabriqué, on peut également sélectionner un contenu culturel spécifique ainsi que cibler un degré précis de difficulté. Il va sans dire que le scénario pédagogique est à la fois un moyen pour l'apprenant de s'entraîner à certains types d'exercices à l'écrit (rédaction de lettres, de rapports...) et un moyen pour lui de véritablement prendre en charge une activité orale (tel que le débat ou la simulation de réunion) sans l'intervention directe de l'enseignant; les phases de réception d'informations sont autant d'occasions pour l'apprenant de gérer un travail en autonomie complète. Pour l'enseignant, le scénario pédagogique représente un outil adaptable qui répond non seulement à de multiples besoins langagiers à divers niveaux de compétence, mais aussi à des besoins de contenu préprofessionnel du public cible. Dans ce contexte, les nouvelles technologies éducatives - que nous appellerons désormais les nouvelles technologies de formation, d'information 
et de communication (terminologie empruntée à Petitgirard 1996) - viennent enrichir les documents-ressources dont le concepteur se servira pour développer une mise en situation donnée.

\section{Utilisation des nouvelles technologies de formation, d'information et de communication dans l'exploitation du scénario pédagogique}

Un des inconvénients des NTFIC réside dans leur intégration efficace au sein d'une séquence pédagogique. Certes, les didacticiels par exemple fournissent un support nouveau pour travailler le vocabulaire, la grammaire, etc., et il existe de plus en plus de CD-ROM interactifs permettant à l'apprenant de s'entraîner à la production orale. Mais que faire des encyclopédies multimédias, qui sont de beaux outils mais qui n'ont pas d'applications pédagogiques directes ? L'hypertexte, aussi, offre l'avantage de permettre à l'étudiant de « naviguer » dans un texte pour en élucider des éléments non compris, mais un travail sur un texte ne constitue pas forcément un objectif pédagogique en soi. Quant à l'Internet, il représente une mine de ressources mais doit être manipulé avec précaution si l'on veut éviter que les apprenants ne passent leur temps à faire du "net surfing "; là encore un objectif précis d'intégration est nécessaire pour justifier l'intérêt pédagogique.

Le scénario répond à ces exigences de "ciblage» par l'emboîtement de taches complémentaires au service d'un objectif général. Les nouvelles technologies de formation servent à l'entraînement aux compétences linguistiques spécifiques, par exemple le CD-ROM interactif NEGO WORLD permet de travailler la présentation d'une entreprise, une situation précise de négociation... Les nouvelles technologies d'information (encyclopédies multimédias, le Web) s'intègrent facilement dans les phases dédiées à la réception d'informations; leur utilisation peut être « didactisée » en créant des grilles de compréhension qui guideront les recherches de l'apprenant. Pour le Web il est fortement conseillé de diriger l'apprenant sur un service spécifique et de lui fournir une grille de guidage (ceci n'empêche pas de récupérer des documents ainsi trouvés en les imprimant). L'utilisation pédagogique du courrier électronique se justifie pour les phases III et IV dans le cadre d'un scénario interlangues entre apprenants dans des établissements géographiquement éloignés. Les nouvelles technologies de communication peuvent être utilisées pour la réalisation de la phase $\mathrm{V}$ : toujours dans le cadre d'un scénario interlangues, la simulation de la situation choisie serait faite par visioconférence ou par conférence téléphonique. Ces exemples ne tiennent pas compte de l'utilisation du laboratoire de langues «nouvelle génération » qui offre de nombreuses possibilités pédagogiques dans la préparation du scénario (un tableau comparatif des supports classiques utilisés phase par phase et les supports NTFIC qui peuvent être substitués se trouve en annexe 1).

En somme le scénario pédagogique est l'outil idéal pour intégrer une diversité de supports nouveaux et de contenus dans un contexte clairement délimité tout en favorisant l'autonomie de l'apprenant et l'interactivité pendant le cours. 


\section{Expérimentations réalisées}

18 Nous allons maintenant examiner deux scénarios pédagogiques utilisés avec deux groupes différents à la Maison des langues et des cultures de l'Université Grenoble 3, structure qui bénéficie d'un équipement à la pointe de la technologie : le Centre d'AutoApprentissage dispose de 31 postes multimédias, 28 postes audio et 11 postes vidéo. Il y a également trois laboratoires de type classique (audio/vidéo) - mais de nouvelle génération car pilotés par informatique - et un laboratoire de langues multimédia. C'est face à de telles machines que l'on se rend compte très vite du peu de matériel pédagogique disponible dans le commerce. L'idée des scénarios présentés était donc de tirer profit des installations tout en respectant les exigences des différentes phases déjà décrites.

Une première utilisation timide fut le scénario Affirmative Action in the United States créé pour des étudiants en maîtrise "Juristes-économistes trilingues" (MST-JET) dans un cours appelé «Pratique de la langue ». Ces étudiants sont de bon niveau, certains ayant séjourné à l'étranger un an ou plus. L'objectif pédagogique de ce scénario était l'intégration d'un contenu culturel spécifique et l'introduction de l'interactivité entre apprenants par le biais de la fonction « réseau téléphonique » du laboratoire de langues. La mise en situation (toujours rédigée en français) est comme suit :

Vous venez d'être sélectionné(e) pour participer à un grand débat télévisé qui sera diffusé à une heure de grande écoute par la chaîne CBS.

Le sujet du débat est :

« Does Affirmative Action mean No White Men Need Apply?»

Vous prendrez l'identité de la personne indiquée plus bas, et vous défendrez ses opinions ; vous pouvez aussi les développer à l'aide d'informations que vous aurez recueillies dans les différentes phases de préparation au débat à savoir :

Phase I

Vous allez téléphoner rapidement à d'autres personnes invitées au débat afin de déterminer leurs opinions respectives; ceci vous aidera à préparer votre contreargumentation lors du débat en Phase V.

Phase II

Recueil d'informations à partir d'une source audio.

Le document que vous allez écouter est la bande sonore d'un reportage de CBS News intitulé "Bernard Goldberg's America", réalisé à la suite du rassemblement d'hommes noirs (Million Man March) qui a eu lieu à Washington le 16 octobre 1995.

Phase III

Lecture de deux articles de presse « Is the U.S. ready to go colour-blind? » (Vocable 31.08.95) et « Death by Judges? » (The Economist 17.06.95)

La grille de guidage vous aidera à sélectionner les éléments les plus pertinents concernant les aspects juridiques, économiques et politiques du problème.

Phase IV

Vous écrirez un rapport de 250 mots dans lequel vous ferez la synthèse des avantages et des inconvénients de la mise en œuvre d'Affirmative Action.

Phase V

Participation au débat télévisé.

Dans un premier temps chaque participant (ou groupe de participants) fera une présentation courte mais structurée de son point de vue personnel. Dans un deuxième temps, lors d'une discussion ouverte, chacun répondra aux arguments des autres par des contre-arguments motivés.

Déroulement du travail :

Séance 1 Phase d'accueil : 15 minutes 
Phase I : 30 minutes

Phase II et III : 1 heure 30 minutes (gestion libre des tâches)

Séance 2 Phase $V: 45$ minutes maximum

Le travail de la phase IV est à rendre la semaine suivant la séance 2

Voici votre identité (photo d'une personne avec nom, âge, profession et 2 ou 3

phrases résumant son point de vue sur la question)

On remarquera d'abord que l'ordre des phases a changé (c'est-à-dire la phase I devient la première phase d'interactivité à la place de la phase III), et ceci pour une raison purement technique : on ne peut pas gérer en même temps le réseau téléphonique et l'écoute libre du document audio de la phase III. De toute façon il est préférable de limiter la phase I dans le temps pour éviter une dégradation de l'ambiance du cours. Même si dans l'ensemble les étudiants ont pris plaisir à jouer le jeu, l'écoute discrète des échanges a permis le maintien de l'anglais dans les conversations. De toute façon, ce risque de dérapage devient peu important lorsque l'on le compare à la dynamique de production orale observée pendant le déroulement de cette phase.

21 Une deuxième expérience plus complexe a été faite dans le cadre d'un séminaire Langues et cultures sur le thème du discours politique et la campagne électorale aux États-Unis. Ce cours s'adresse à un public interuniversitaire (mélange d'étudiants spécialistes et nonspécialistes) de niveau relativement homogène, puisqu'il y a eu test de langue à l'entrée.

L'objectif pédagogique du scénario Choosing a candidate était double: d'une part, permettre aux étudiants de mieux comprendre l'enjeu des élections primaires du parti républicain et de leur faire vivre la situation " de l'intérieur » en leur attribuant un rôle d'électeur; d'autre part, utiliser les installations du Centre d'Auto-Apprentissage (postes multimédia, vidéo et audio) pour l'accomplissement des trois phases de recueil d'informations et ce en laissant l'apprenant maître de ses choix en ce qui concerne le nombre et le type de documents-ressources consultés.

La mise en situation est comme suit :

Vous êtes un électeur de l'État d'Iowa qui va voter dans l'élection primaire. Vous devez choisir entre les nombreux candidats républicains; le choix est difficile, et pour pouvoir participer à émission de radio (diffusion nationale) sur les motivations de votre soutien pour tel ou tel autre candidat, il faut accomplir les tâches suivantes

Phase I :

Vous devez vous créer une identité politique.

Pour ce faire, vous disposez de trois documents concernant les tendances politiques aux États-Unis dans le contexte des élections présidentielles :

- vous remplirez le questionnaire « Who are you?»;

- vous étudierez les sondages ;

- vous choisirez, parmi les profils politiques proposés, celui qui vous décrit le mieux et qui correspond aux réponses que vous avez données dans le questionnaire.

Phase II :

Vous allez écouter ou visionner un document qui présente 4 candidats républicains potentiels ainsi que Bill Clinton; vous devez recueillir toutes les informations qui vous permettront de connaître au mieux chacun d'entre eux. ${ }^{1}$

Phase III :

Vous allez écouter ou visionner un document qui présente des électeurs de l'État d'Iowa qui vont voter dans l'élection primaire. Vous devez recueillir toutes les informations qui vous permettront de connaître au mieux chacun d'entre eux ainsi que leurs opinions concernant les différents candidats. Ces informations devront vous aider à créer une identité que vous assumerez pendant la Phase V. 


\begin{abstract}
Phase IV :
Vous allez rédiger un tract vantant les mérites du candidat de votre choix, en tenant compte du programme politique de celui-ci et de vos propres convictions à son égard. Ce tract sera composé au minimum d'un texte et d'un slogan.

Phase V :

Vous allez participer à une émission de radio (diffusion nationale) pendant laquelle vous annoncerez pour qui vous avez voté et le pourquoi de ce vote. Vous participerez ensuite à un débat ouvert pendant lequel chaque participant tentera de convaincre les autres du bien-fondé de son propre choix.
\end{abstract}

Le déroulement des différentes phases préliminaires à la phase $\mathrm{V}$ était organisé en "chasse aux informations" (information hunt) : sur deux postes multimédias étaient installés des CD-ROM encyclopédiques ${ }^{2}$ et il a été indiqué aux étudiants que les informations générales sur le système électoral américain étaient disponibles s'ils en ressentaient le besoin ; sur 4 postes vidéo, ils pouvaient visionner des montages différents correspondant aux informations à recueillir pour les phases II et III ; sur les postes audio, les bandes sonores des documents vidéo étaient proposées à l'écoute pour les étudiants souhaitant se passer des images et se concentrer sur le contenu. Le temps imparti pour les trois premières phases était de deux heures en autonomie libre, mais en réalité certains étudiants du groupe sont revenus poursuivre ce travail entre deux séances ; l'étendue des informations recueillies variait donc suivant le niveau de compétence de chaque étudiant et le nombre d'heures consacrées à la réalisation des tâches en phase II et en phase III.

La séance suivante a servi à faire une mise en commun de la masse d'informations que les étudiants avaient en leur possession et à déterminer quel candidat chaque étudiant avait choisi et pourquoi. En fait il s'agissait de préparer de façon informelle et informative (le rôle de l'enseignant étant d'éclaircir les zones d'ombre et de recentrer les débats) le déroulement de la phase $\mathrm{V}$ qui aurait lieu dans le studio d'enregistrement ; mis dans une situation inhabituelle, les étudiants éprouvaient le besoin de faire une mini-simulation préalable, sans pour autant faire une véritable répétition du débat à suivre.

5 L'objectif de l'enregistrement dans un studio de type professionnel était double : simuler les conditions réelles d'une émission de radio (casques, micros, petite salle insonorisée...) et obtenir des échantillons de production orale dans une situation de présentation/ argumentation. Après montage (qui ne veut pas dire truquage), la bande sonore a fait l'objet d'un cours pendant lequel les étudiants ont analysé les divers aspects de leurs propres performances, à savoir l'étude de la compétence linguistique (grammaire, lexique et phonologie), la réactivation des contenus, la mise en œuvre de stratégies de compensation (reformulations, paraphrases, demandes d'aide...) et la gestion du débat (questions posées, relances, contre-arguments, respect du temps de parole, etc.).

Pour terminer, on pourrait arguer qu'une telle organisation demande une préparation technique relativement longue (enregistrements d'émissions télévisées puis montages thématiques en particulier) et que la campagne des élections primaires aux États-Unis est un sujet éphémère. À cela, on répondra tout simplement qu'à partir de ces mêmes documents il sera possible dans six mois (lors des présidentielles) ou dans deux ans (lors des mid-term elections) de recréer une nouvelle séquence pédagogique qui simule les conditions électorales et qui débouche sur une étude du discours politique. Il va sans dire qu'un scénario à ciblage culturel spécifique de ce type exige son intégration dans un cours plus vaste qui traite de multiples domaines civilisationnels que nous ne développerons pas ici. 


\section{Projets}

27 Jusqu'ici nous avons peu parlé de l'utilisation de l'Internet dans le scénario pédagogique, car notre expérience reste limitée pour l'instant. Cependant l'exploitation idéale des « autoroutes de l'information » trouvera sa réalisation dans la construction et l'utilisation du scénario interlangues entre établissements géographiquement éloignés; notre premier projet aura lieu entre un groupe d'étudiants en F.L.E. (français langue étrangère) d'une université nord-américaine et un groupe d'anglais de la Maison des langues et des cultures. L'idée est de créer un scénario unique pour les deux langues: les uns exécuteront ce scénario en français, les autres en anglais. La phase III sera le point de rencontre et d'échange dans les deux langues par conférence téléphonique, le courrier électronique peut être le véhicule de la phase IV et on imagine aisément la mise en place d'une visioconférence pour la phase V. Il s'agit bien entendu d'un projet qui reste pour l'instant dans un stade embryonnaire car il faut résoudre certains problèmes concernant la double réalisation des phases d'interactivité afin que les deux groupes s'entraînent à l'utilisation des langues cibles respectives. Néanmoins l'Internet ouvre de vastes possibilités d'internationalisation du scénario pédagogique.

En guise de conclusion, nous devons rappeler au lecteur non averti l'incontournable Loi de Murphy ${ }^{3}$ présente dans toute situation où le pédagogue rend la technologie indispensable à l'accomplissement de son cours : personne n'est à l'abri d'une simple panne d'électricité qui anéantira en quelques secondes des heures de travail préparatoire, pour ne citer que l'exemple le plus évident. Certes, les nouvelles technologies ont révolutionné, et révolutionneront encore, l'enseignement des langues; utilisées à bon escient elles viendront enrichir les contenus que nous offrons à nos apprenants. Mais elles ne remplaceront jamais la démarche pédagogique, qui reste, et nous dirons heureusement, l'apanage des humbles mortels que nous sommes.

\section{BIBLIOGRAPHIE}

Isani, Shaeda, Micheline Herino et Donna Andreolle. 1996. « Méthodologie de Création de Scénarii D.C.L. ». Rapport non publié, Université Grenoble 3.

Petitgirard, Jean-Yves. 1996. « Le multimédia ». Les Langues Modernes 1, 6-13.

\section{ANNEXES}

Annexe 1 : Utilisation des nouvelles technologies de formation, d'information et de communication dans l'exploitation du scénario pédagogique

Phase I - recueil d'informations 


\begin{tabular}{|l|l|}
\hline Supports classiques & Supports N.T.F.I.C. \\
\hline Textes imprimés (brochures, articles, publicités, plans...) & $\begin{array}{l}\text { CD-ROM de type encyclopédique } \\
\text { Hypertexte } \\
\text { Internet (services du Web) }\end{array}$ \\
\hline
\end{tabular}

Phase II - recueil d'informations

\begin{tabular}{|l|l|}
\hline Supports classiques & Supports N.T.F.I.C. \\
\hline $\begin{array}{l}\text { Documents audiovisuels } \\
\text { (bande sonore seule ou vidéo) } \\
\text { Laboratoire de langues } \\
\text { «ancienne génération» }\end{array}$ & $\begin{array}{l}\text { Labuments audiovisuels + CD-ROM utilisés en... } \\
\text { Laboratoire de langues «nouvelle génération » (fonctions du } \\
\text { type-conférence en groupe/réseau téléphonique...) }\end{array}$ \\
\hline
\end{tabular}

Phase III - Interactivité

\begin{tabular}{|l|l|}
\hline Supports classiques & Supports N.T.F.I.C. \\
\hline $\begin{array}{l}\text { Face-à-face interlocuteur/ } \\
\text { apprenant ou candidat }\end{array}$ & $\begin{array}{l}\text { Laboratoire de langues «nouvelle génération » (fonctions du } \\
\text { type-conférence en groupe/réseau téléphonique...) } \\
\text { E-Mail (scénario interlangues) }\end{array}$ \\
\hline
\end{tabular}

Phase IV - travail de synthèse (rédaction)

\begin{tabular}{|l|l|}
\hline Supports classiques & Supports N.T.F.I.C. \\
\hline Travail sur papier & Traitement informatique \\
& E-Mail (scénario interlangues) \\
\hline
\end{tabular}

Phase V - Interactivité (présentation/argumentation)

\begin{tabular}{|l|l|}
\hline Supports classiques & Supports N.T.F.I.C. \\
\hline Face-à-face interlocuteur/apprenant ou candidat & Conférence téléphonique \\
Visioconférence
\end{tabular}

Annexe 2 : Exemples de grilles de guidage pour le scénario pédagogique Choosing a Candidate 
Phase II

Vous allez écouter ou visionner un document qui présente 5 candidats potentiels de l'élection présidentielle de 1996. Vous devez recueillir toutes les informations qui vous permettront de connaître au mieux chacun d'entre eux.

\begin{tabular}{|c|c|c|c|c|}
\hline & $\begin{array}{l}\text { "Vital statistics" } \\
\text { (Political \& personal } \\
\text { info) }\end{array}$ & $\begin{array}{l}\text { Examples } \\
\text { discourse } \\
\text { (key words and } \\
\text { phrases) }\end{array}$ & $\begin{array}{l}\text { Political platform } \\
\text { for this } \\
\text { election }\end{array}$ & $\begin{array}{l}\text { Criticism aimed at } \\
\text { him } \\
\text { /at his campaign }\end{array}$ \\
\hline Bob Dole & & & & \\
\hline $\begin{array}{l}\text { Lamar } \\
\text { Alexande }\end{array}$ & & & & \\
\hline $\begin{array}{l}\text { Steve } \\
\text { Forbes }\end{array}$ & & & & \\
\hline $\begin{array}{l}\text { Bill } \\
\text { Clinton }\end{array}$ & & & & \\
\hline $\begin{array}{l}\text { Richard } \\
\text { Lugar }\end{array}$ & & & & \\
\hline
\end{tabular}

Phase III

Vous allez écouter ou visionner un document qui présente des électeurs de l'État d'Iowa qui vont voter dans l'élection primaire. Vous devez recueillir toutes les informations qui vous permettront de connaître au mieux chacun d'entre eux ainsi que leurs opinions concernant les différents candidats. Ces informations devront vous aider à créer une identité que vous assumerez pendant la Phase V.

\begin{tabular}{|l|l|l|l|l|}
\hline $\begin{array}{l}\text { Name, } \\
\text { gender }\end{array}$ & $\begin{array}{l}\text { Professional } \\
\text { background and } \\
\text { personal information }\end{array}$ & $\begin{array}{l}\text { Key issue of the } \\
\text { campaign for this } \\
\text { person }\end{array}$ & $\begin{array}{l}\text { Who this person } \\
\text { will vote for and } \\
\text { why }\end{array}$ & $\begin{array}{l}\text { Other opinions on } \\
\text { politicsor on } \\
\text { politicians }\end{array}$ \\
\hline & & & & \\
\hline
\end{tabular}

\section{NOTES}

1. Voir grilles de guidage en annexe 2 .

2. Il s'agit de Grolier's Multimedia Encyclopedia, Compton's Interactive Encyclopedia et Encarta.

3. Murphy's Law : «If anything can go wrong, it will!». 


\section{RÉSUMÉS}

L'enseignement des langues subit actuellement une mutation due à l'explosion des nouvelles technologies de formation, d'information et de communication qui bouleversent nos « attitudes » pédagogiques dans la salle de classe. Cet article présente le scénario de tâches complémentaires et son utilisation dans le cadre d'un séminaire langues et cultures sur le discours politique aux États-Unis, ainsi que le rôle de divers outils multimédias dans la réalisation des différentes phases de travail proposées aux apprenants.

The ever-increasing presence of new educational materials in the multimedia environment is reshaping teaching attitudes in the language classroom. This paper analyzes the ways in which it is possible to introduce tools such as CD-ROMs, multimedia language labs and the Internet into an interactive teaching situation through the use of "teaching scenarios". After a brief presentation of the pedagogical philosophy behind the creation of such scenarios, several examples of classroom experimentation and possible future development are examined.

INDEX

Mots-clés : attitude pédagogique, discours politique, langue de spécialité, multimédia, nouvelles technologies, tâche

Keywords : learning task, LSP, new technologies, pedagogical attitude, political discourse

\section{AUTEUR}

\section{DONNA ANDREOLLE}

Donna Andreolle est professeur agrégée d'anglais à la Maison des langues et des cultures de l'Université Stendhal Grenoble 3. dandreolle@gmail.com 